\title{
A New Electric Field in Asymmetric Magnetic Reconnection
}

\author{
K. Malakit, ${ }^{1,2}$ M. A. Shay, ${ }^{2}$ P. A. Cassak, ${ }^{3}$ and D. Ruffolo ${ }^{1,4}$ \\ ${ }^{1}$ Department of Physics, Mahidol University, Bangkok 10400, Thailand \\ ${ }^{2}$ Department of Physics and Astronomy, University of Delaware, Newark, Delaware 19716, USA \\ ${ }^{3}$ Department of Physics, West Virginia University, Morgantown, West Virginia 26506, USA \\ ${ }^{4}$ Thailand Center of Excellence in Physics, CHE, \\ Ministry of Education, Bangkok 10400, Thailand
}

(Dated: September 28, 2018)

\begin{abstract}
We present a theory and numerical evidence for the existence of a previously unexplored inplane electric field in collisionless asymmetric magnetic reconnection. This electric field, dubbed the "Larmor electric field," is associated with finite Larmor radius effects and is distinct from the known Hall electric field. Potentially, it could be an important indicator for the upcoming Magnetospheric Multiscale (MMS) mission to locate reconnection sites as we expect it to appear on the magnetospheric side, pointing Earthward, at the dayside magnetopause reconnection site.
\end{abstract}

PACS numbers: 52.35.Vd, 94.30.cp

\section{INTRODUCTION}

Magnetic reconnection efficiently converts magnetic energy into heat and flow energy of particles in plasmas (e.g., [1]). It occurs when small-scale dissipation permits an electric field that breaks the frozen-in condition and allows magnetic field lines to change topology. It naturally arises in many locations in the Earth's magnetosphere. The dissipation region is often difficult to measure in naturally occurring settings because it is small compared to global scales. The primary objective of the upcoming Magnetospheric MultiScale (MMS) mission is to study the properties of the dissipation region in reconnection [2, 3]. Therefore, it is of critical importance to determine the observational signatures of magnetic reconnection near the dissipation region.

One signature of the dissipation region in collisionless reconnection is due to the Hall effect. Since ions and electrons have different Larmor radii due to their different masses, they undergo different bulk motion within small distances from the reconnection site. This sets up in-plane Hall currents, which are associated with outof-plane magnetic fields and in-plane electric fields [47]. During symmetric reconnection as is most often studied theoretically and numerically, the Hall magnetic field is quadrupolar and the Hall electric field is bipolar. This signature of collisionless reconnection has been observed using satellite observations at the dayside magnetopause [8-12]. Note that observations of the quadrupolar Hall magnetic and bipolar Hall electric field are rare at the dayside magnetopause [13] because reconnection normally has asymmetric inflow, which changes the structure of the Hall fields [14, 15]. In some cases, the asymmetry causes the Hall magnetic and electric fields to become so skewed that they become bipolar and unipolar, respectively [16-19]. These skewed Hall structures have also been observed [16, 18, 20].

In this paper, we argue for the existence of an in-plane electric field that was not previously discussed, which appears in asymmetric reconnection and can be used as an observational signature of the dissipation region. This electric field is caused by finite Larmor radius effects, so we dub it the "Larmor electric field." The electric field structure is located in the inflow region of the dissipation region, just upstream of the Hall electric field structure, with its direction pointing away from the X-line. We present a physical argument for its existence and show it is consistent with the results of fully-kinetic particle-incell (PIC) simulations.

Under normal dayside reconnection conditions, we show that the Larmor electric field should be present on the magnetospheric side of the X-line pointing Earthward with a magnitude large enough to be measurable with spacecraft. Therefore, its existence can be useful for locating dissipation regions in the MMS mission.

\section{THEORY}

To understand where the Larmor electric field comes from, consider the structure of the dissipation region. In symmetric anti-parallel magnetic reconnection, the Xline and stagnation point are located at the due center of the dissipation region. When there are asymmetries in the magnetic field $B$ and/or plasma density $n$, this is no longer the case [21]. The X-line location is determined by energy conservation, while the stagnation point is determined by mass conservation. In particular, the stagnation point location is given by 21]

$$
\frac{\delta_{S 2}}{\delta_{S 1}} \sim \frac{n_{2} B_{1}}{n_{1} B_{2}}
$$

where $\delta_{S}$ is the distance from the stagnation point to one edge of the dissipation region and the subscripts " 1 " and " 2 " denote the inflow side of interest. The stagnation point is offset towards the side with smaller $n / B$. 
The basic picture of the dissipation region structure is sketched in Fig. 1. The sketch is roughly for typical magnetopause inflow conditions (density variation by a factor of 10, magnetic field variation by a factor of 2 ), with the magnetosheath and magnetosphere as Populations 1 and 2, respectively. The low magnetospheric density implies that the stagnation point is shifted very close to the Population 2 side of the dissipation region. The inflowing Population 2 plasma is therefore constrained in a basic fluid sense to turn the corner sharply and flow outwards. Once magnetic field lines reconnect, however, the two populations begin to mix along the magnetic field lines.

A question arises in the context of a kinetic plasma. The Larmor radii of the two populations are shown as circles in the inflow regions of Fig. 1, If the Larmor radius $\rho_{i 2}$ of Population 2 ions significantly exceeds $\delta_{S 2}$, it is not clear how the basic dissipation region structure is maintained. However, the scaling of basic reconnection properties in kinetic simulations are consistent with the scaling theory based on fluid conservation laws [19], so one does not expect a breakdown of the conservation laws. Instead, we conclude that an electric field upstream of the dissipation region must appear to prevent Population 2 ions from crossing the stagnation point. This Larmor electric field arises due to the premature leakage of a small percentage of Population 2 ions into the dissipation region, which creates charge separation.

We now make this more quantitative. From the above argument for its existence, the spatial extent $\delta_{E \text {,Larmor }}$ of the Larmor electric field should scale with the Larmor radius $\rho_{i 2}$ of Population 2 ions,

$$
\delta_{E, \text { Larmor }} \sim \rho_{i 2}
$$

The magnitude of the electric field $E_{\text {Larmor }}$ can be estimated by noting that the premature leakage of Population 2 ions into the dissipation region will occur until the potential barrier for an ion due to the Larmor electric field is comparable to the average kinetic energy per charge:

$$
E_{\text {Larmor }} \sim \frac{k_{B} T_{i 2}}{e \rho_{i 2}},
$$

where $T_{i 2}$ is the temperature of Population 2 ions, $e$ is the ion charge, and $k_{B}$ is Boltzmann's constant. Note that the energy density of this Larmor electric field is negligible compared to the thermal energy density of the Population 2 plasma. Finally, the Larmor electric field should only exist when

$$
\rho_{i 2}>\delta_{S 2}
$$

In the remainder of this paper, we present evidence from simulations for the Larmor electric field and discuss implications for observations in the Earth's magnetosphere.

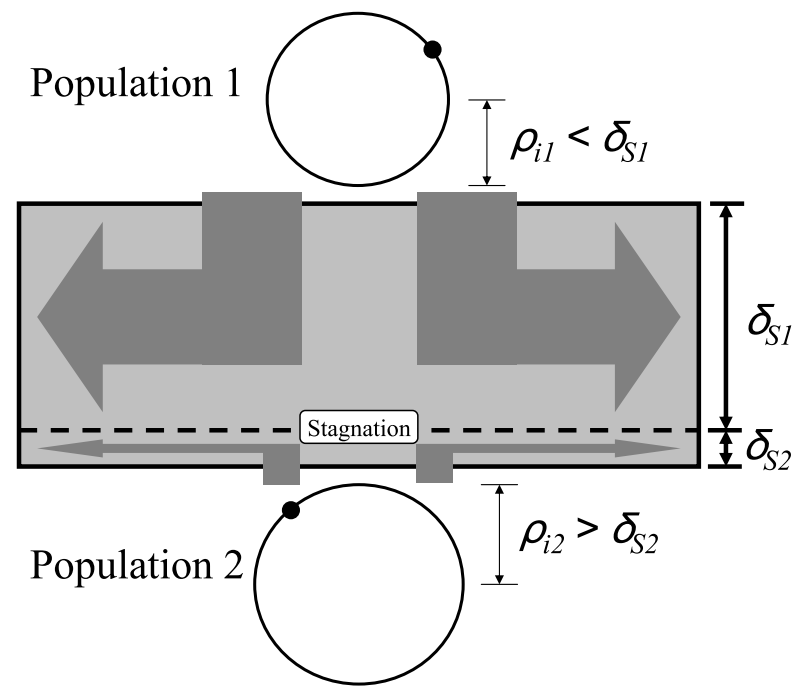

FIG. 1. Schematic of the flow structure of the dissipation region during asymmetric reconnection for characteristic dayside-magnetosphere parameters in a kinetic plasma.

\section{SIMULATIONS}

We use the parallel particle-in-cell (PIC) code P3D 22] to perform simulations in 2.5 dimensions of collisionless anti-parallel asymmetric reconnection. In the simulations, magnetic field strengths and particle number densities are normalized to arbitrary values $B_{0}$ and $n_{0}$, respectively. Lengths are normalized to the ion inertial length $d_{i 0}=c / \omega_{p i}$ at the reference density. Time is normalized to the ion cyclotron time $\Omega_{c i 0}^{-1}=$ $\left(e B_{0} / m_{i} c\right)^{-1}$. Speeds are normalized to the Alfvén speed $c_{A 0}=B_{0} /\left(4 \pi m_{i} n_{0}\right)^{1 / 2}$. Electric fields and temperatures are normalized to $E_{0}=c_{A 0} B_{0} / c$ and $T_{0}=m_{i} c_{A 0}^{2} / k_{B}$, respectively.

Simulations are performed in a periodic domain of size $L_{x} \times L_{y}=204.8 \times 102.4$ with a grid scale $\Delta x=\Delta y$ of 0.05 . The time step $\Delta t$ is $0.0025,0.005$, or 0.01 . The normalization density $n_{0}$ is represented by a number of particles per grid cell, or ppg, which ranges from 50 to 200. The ion to electron mass ratio for all but Run $1^{*}$ is $m_{i} / m_{e}=25$ and the speed of light $c$ is $15 c_{A 0}$.

The initial conditions are a double asymmetric current sheet (see Ref. [19] for more details). A small magnetic perturbation is used to initiate reconnection. Each simulation is evolved until reconnection reaches a steady state. The parameters (magnetic fields, densities, electron temperatures and ion temperatures on either side of the dissipation region) for each simulation are shown in Table I] 
TABLE I. Parameters for the present simulation study. Subscripts "1" and "2" refer to the two upstream sides of the dissipation region. The values give the magnetic field strengths $B$, number densities $n$, electron temperatures $T_{e}$ and ion temperatures $T_{i}$. For each run, the width of the Larmor electric field $\delta_{E, \text { Larmor }}$ and its magnitude $E_{\text {Larmor }}$ are also provided.

\begin{tabular}{c|cc|cc|cccc|cc}
\hline \hline Run & $B_{1}$ & $B_{2}$ & $n_{1}$ & $n_{2}$ & $T_{e 1}$ & $T_{i 1}$ & $T_{e 2}$ & $T_{i 2}$ & $\delta_{E, \text { Larmor }}$ & $E_{\text {Larmor }}$ \\
\hline 1 & 1.0 & 2.0 & 1.0 & 0.1 & 0.67 & 1.33 & 1.67 & 3.33 & 3.80 & 1.09 \\
$1^{*}$ & 1.0 & 2.0 & 1.0 & 0.1 & 0.67 & 1.33 & 1.67 & 3.33 & 3.50 & 0.86 \\
2 & 1.0 & 2.0 & 1.0 & 0.25 & 0.67 & 1.33 & 0.67 & 1.33 & 2.50 & 0.44 \\
3 & 1.0 & 1.0 & 1.0 & 0.1 & 0.67 & 1.33 & 6.67 & 13.33 & 7.80 & 1.31 \\
4 & 2.0 & 2.0 & 1.0 & 0.1 & 0.67 & 1.33 & 6.67 & 13.33 & 5.70 & 2.13 \\
5 & 1.0 & 1.0 & 1.0 & 0.5 & 0.67 & 1.33 & 1.33 & 2.67 & 4.15 & 0.19 \\
6 & 1.5 & 1.0 & 1.0 & 0.1 & 0.67 & 1.33 & 8.75 & 17.50 & 8.10 & 1.25 \\
7 & 1.5 & 1.0 & 1.0 & 0.2 & 0.67 & 1.33 & 4.38 & 8.75 & 6.90 & 0.79 \\
8 & 1.5 & 1.0 & 1.0 & 0.44 & 0.67 & 1.33 & 1.99 & 3.98 & 5.35 & 0.37 \\
9 & 2.0 & 1.0 & 1.0 & 0.25 & 0.67 & 1.33 & 4.67 & 9.33 & 7.40 & 0.58 \\
10 & 3.0 & 1.0 & 1.0 & 0.11 & 0.67 & 1.33 & 18.03 & 36.03 & 8.85 & 1.44 \\
11 & 2.0 & 1.0 & 1.0 & 0.44 & 0.67 & 1.33 & 2.65 & 5.30 & 4.20 & 0.42 \\
12 & 3.0 & 1.0 & 1.0 & 0.44 & 0.67 & 1.33 & 4.55 & 9.09 & 4.90 & 0.53 \\
13 & 1.5 & 1.0 & 1.0 & 1.0 & 0.46 & 0.92 & 0.67 & 1.33 & $\mathrm{n} / \mathrm{a}$ & 0.06 \\
14 & 2.0 & 1.0 & 1.0 & 1.0 & 0.17 & 0.33 & 0.67 & 1.33 & $\mathrm{n} / \mathrm{a}$ & 0.04 \\
15 & 2.0 & 1.0 & 0.1 & 0.1 & 0.67 & 1.33 & 5.67 & 11.33 & $\mathrm{n} / \mathrm{a}$ & 0.09 \\
16 & 3.0 & 1.5 & 0.3 & 0.3 & 0.67 & 1.33 & 4.42 & 8.83 & $\mathrm{n} / \mathrm{a}$ & 0.07 \\
17 & 3.0 & 1.0 & 1.0 & 1.0 & 0.67 & 1.33 & 2.00 & 4.00 & $\mathrm{n} / \mathrm{a}$ & 0.09 \\
\hline \hline
\end{tabular}

\section{RESULTS AND DISCUSSION}

We show results from Run 1 as a case study because it shows a particularly clear example of the Larmor electric field and has density and magnetic field variations typical of magnetopause reconnection. The electric field $E_{y}$ in the inflow direction is shown in Fig. 2(a). The blue band on the high density side and the red band are the standard in-plane Hall electric field. The blue band on the side with the stronger magnetic field and lower density is the Larmor electric field. It is most prominent immediately upstream of the dissipation region.

To show that this electric field is not caused by the Hall effect, we present a plot of the contributions to $E_{y}$ from the generalized Ohm's law in Fig. 2(b) in a cut along the vertical dashed line in Fig. 2(a). The vertical solid line marks the X-line. Immediately to the left of the X-line, there is an electric field pointing toward the $\mathrm{X}$-line (the positive peak). This electric field is largely contributed by the Hall term (in blue). For comparison, Fig. 2(c) shows a cut from a symmetric reconnection run, which has the standard bipolar structure of the Hall electric field on both sides of the X-line, and does not display a Larmor electric field.

Further toward smaller $y$ (to the left of the Hall field), there is another electric field structure pointing in the opposite direction to the Hall field. However, the Hall term is very small in this region, so it is not associated with the Hall term. This electric field is the Larmor electric field and points away from the $\mathrm{X}$-line, in contrast to the
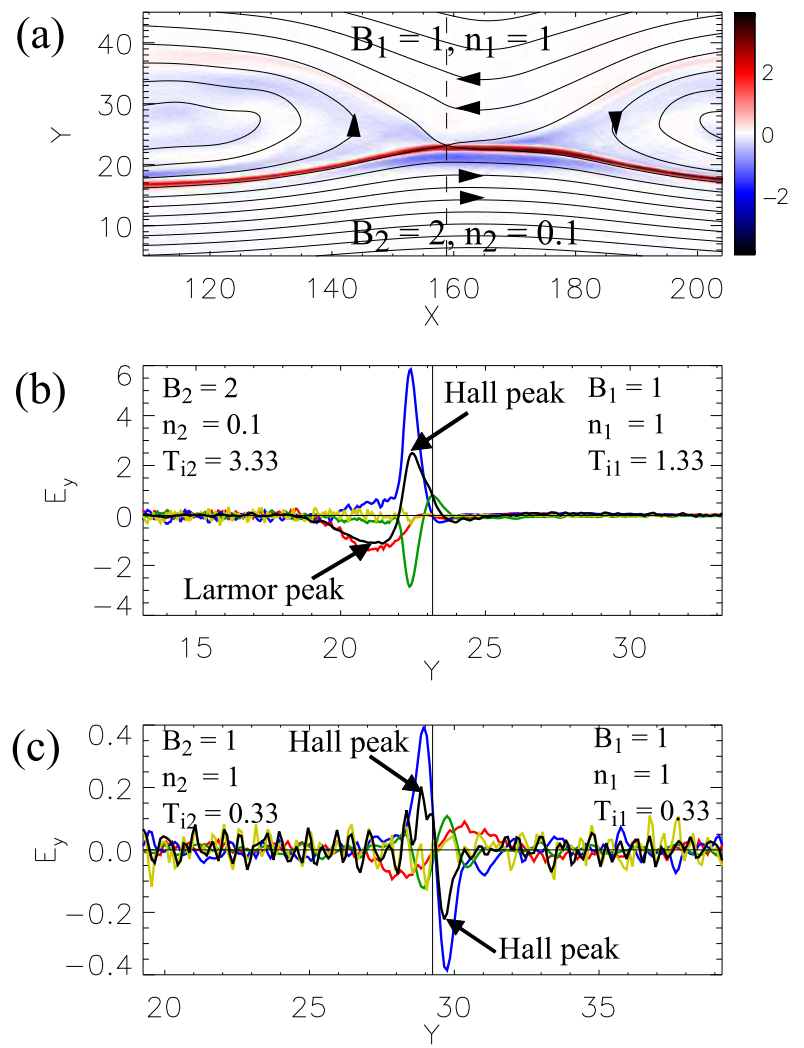

FIG. 2. (a) Plot of the normal electric field $E_{y}$ of Run 1, overplotted with magnetic field lines. Red is positive, blue is negative, and white is zero. (b) Cut of the normal electric field $E_{y}$ (black) and its contributing terms from generalized Ohm's law along the vertical dashed line in (a): the ion convection term $-(1 / c)\left(\mathbf{u}_{\mathbf{i}} \times \mathbf{B}\right)_{y}($ red $)$, Hall term $(1 /$ nec $)(\mathbf{J} \times \mathbf{B})_{y}$ (blue), pressure gradient term $-(1 / n e)\left(\nabla \cdot \mathbf{P}_{\mathbf{e}}\right)_{y}$ (green), and electron inertia term $-\left(m_{e} / e\right)\left(d u_{e} / d t\right)_{y}$ (yellow). (c) Similar cut to (b) but for data from a symmetric run.

Hall electric field which points toward the X-line. (For the parameters of this simulation, the Hall electric field is skewed and has a unipolar, rather than bipolar, electric field.) The Larmor electric field occurs upstream of the dissipation region where the plasma is roughly frozen-in, so it is roughly balanced by the ion convection term in Ohm's law.

The appearance of this electric field is consistent with previous PIC simulations of asymmetric reconnection \{see Fig 10(c) of Ref. 17] and Fig. 4(e) and 10(e) of Ref. [18]\}. It may have been seen in observations s see Fig. 9(h)) of Ref. 23] \}. However, its existence has not been pointed out, and the physics of it has not yet been discussed.

We now consider the parametric dependence of properties of the Larmor electric field. For all simulations in Table I for which the Larmor electric field exists, we measure its spatial extent $\delta_{E \text {,Larmor }}$ in the inflow direction as the distance between the two locations bounding the outward-directed electric field, i.e., where $E_{y}$ 
changes sign [at around $y=18.5$ and 22 in the example in Fig. 2(b)]. The results are shown in Fig. 3(a),

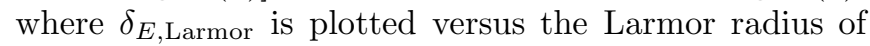
the upstream ions $\rho_{i 2}$, which is evaluated using the ion temperature and magnetic field $10 d_{i 0}$ upstream of the $\mathrm{X}$-line. A clear correlation between the two length scales is apparent, in agreement with Eq. (2).

The magnitude of $E_{\text {Larmor }}$ is measured where the electric field peaks in the direction away from the $\mathrm{X}$-line. For simulations in which the Larmor electric field exists, the results are plotted in Fig. 3. (b), with the predicted Larmor field strength $k_{B} T_{i 2} / e \rho_{i 2}$ from Eq. (3) on the horizontal axis. Scaling according to Eq. (3) is observed.

This result suggests that the energy gained/lost by an ion that crosses the electric field structure is only in the order of the thermal energy of an ion from Population 2 $k_{B} T_{i 2}$. Hence, this electric field does not significantly participate in particle acceleration.

Finally, we note that there are five simulations for which the Larmor electric field does not arise. From Eq. (4), we expect the Larmor electric field to exist when $\rho_{i 2}$ exceeds the distance $\delta_{S 2}$ from the stagnation point to the edge of the dissipation region on the side of the dissipation region with the Larmor electric field.

To test this hypothesis, we must find $\delta_{S 2}$. This is difficult to measure directly from the simulations due to the noise inherent in PIC simulations, so we appeal to asymmetric reconnection theory. Equation (1) gives the relative length scales, so we need the total thickness of the dissipation region. For collisionless asymmetric reconnection, the half thickness of the dissipation region $\delta$ was predicted to be [24]

$$
\delta \sim \frac{B_{1}+B_{2}}{2 \sqrt{B_{1} B_{2}}}\left[\left(\frac{m_{i}^{2} c^{2}}{4 \pi e^{2}}\right)\left(\frac{B_{1}+B_{2}}{m_{i}\left(n_{1} B_{2}+n_{2} B_{1}\right)}\right)\right]^{1 / 2} .
$$

Using $2 \delta=\delta_{S 1}+\delta_{S 2}$, one finds the predicted absolute size of $\delta_{S 2}$ :

$$
\delta_{S 2} \sim\left[\frac{n_{2} B_{1}}{n_{1} B_{2}+n_{2} B_{1}}\right] 2 \delta .
$$

For comparison with the simulations the values are calculated from the asymptotic values in Table [.

The results are plotted in Fig. 3(c), where the magnitude of the Larmor electric field $E_{\text {Larmor }}$ is plotted versus the ratio $\rho_{i 2} / \delta_{S 2}$. One sees that $E_{\text {Larmor }}$ is non-zero when $\rho_{i 2} / \delta_{S 2} \gtrsim 1$ and is near 0 otherwise, consistent with Eq. (44). Note that the small deviations of $E_{\text {Larmor }}$ from zero for Runs 13-17 are merely due to noise, and

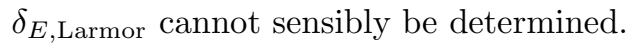

To test the effect of the electron mass, we perform a simulation like Run 1 with an ion to electron mass ratio of 100 instead of 25 , which we call Run $1^{*}$. Comparison between Run 1 and Run $1^{*}$ suggests that the results discussed above are insensitive to the ion to electron mass ratio in the simulations. We therefore expect the results
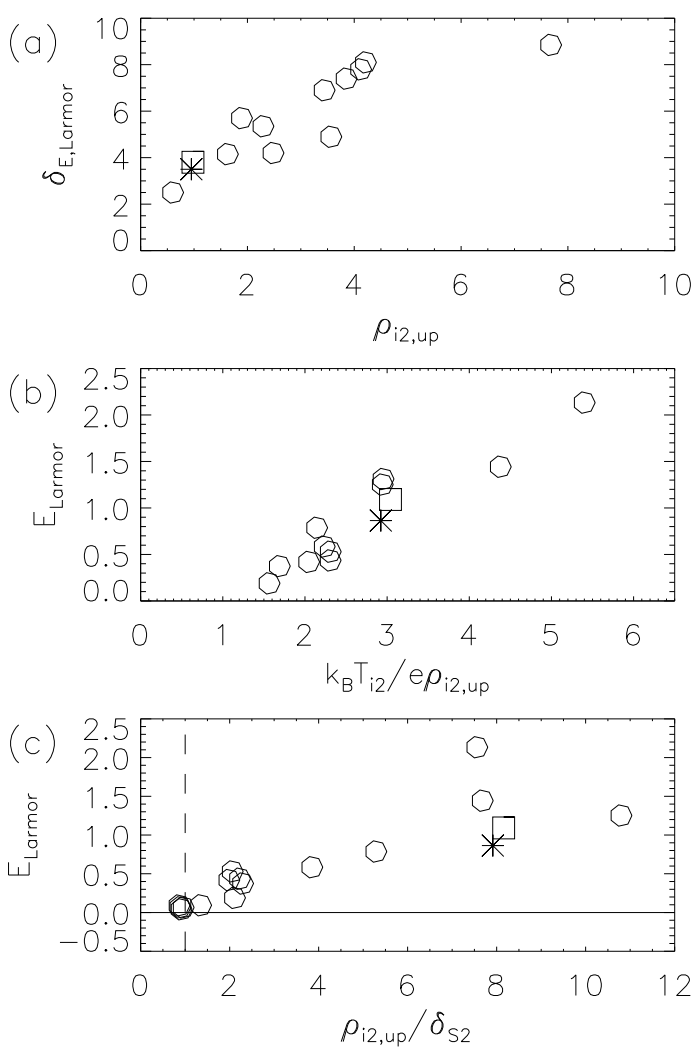

FIG. 3. (a) Thickness of the Larmor electric field $\delta_{E \text {, Larmor }}$ vs. ion Larmor radius $\rho_{i 2}$ on the upstream side with the Larmor electric field. (b) Magnitude of the Larmor field $E_{\text {Larmor }}$ vs. its predicted scaling $k_{B} T_{i 2} / e \rho_{i 2}$. (c) $E_{\text {Larmor }}$ vs. the ratio of $\rho_{i 2}$ to the distance $\delta_{S 2}$ between the stagnation point and the edge of the dissipation region. The square is for Run 1 , and the asterisk is for Run $1^{*}$

to still hold for the actual mass ratio even though it is significantly larger.

\section{APPLICATIONS}

For reconnection at the dayside magnetopause, we argue that the Larmor electric field is expected to be present. Typical magnetic field strengths, densities, and ion temperatures on the magnetosheath side are approximately $20 \mathrm{nT}, 25 \mathrm{~cm}^{-3}$, and $2 \times 10^{6} \mathrm{~K}$, and are $55 \mathrm{nT}, 0.5$ $\mathrm{cm}^{-3}$, and $20 \times 10^{6} \mathrm{~K}$ on the magnetospheric side [25]. The distances from the stagnation point to the edges of the dissipation region are calculated using Eqs. (5) and (6) to be about $1 \mathrm{~km}$ on the magnetospheric side and 119 $\mathrm{km}$ on the magnetosheath side. The ion Larmor radii on the magnetospheric and magnetosheath sides are $77 \mathrm{~km}$ and $67 \mathrm{~km}$. Since the ion Larmor radius is expected to be larger than the distance from the stagnation point to the edge of the dissipation region on the magnetospheric side, the Larmor electric field is expected to exist on the magnetospheric side pointing away from the 
$\mathrm{X}$-line, i.e., toward the Earth. The magnitude of the electric field, from Eq. (3), is predicted to be on the order of $20 \mathrm{mV} / \mathrm{m}$. This strength of electric field is easily measurable by spacecraft.

Since the Larmor electric field is localized upstream of the dissipation region, it can be a useful signature to help satellites, such as the MMS mission, identify the dissipation region of reconnection sites before the satellite moves deeper into the dissipation region.

This research was supported by the postdoctoral research sponsorship of Mahidol University (KM), NSF grants ATM-0645271 - Career Award (MAS) and AGS-0953463 (PAC), NASA grants NNX08A083G MMS IDS, NNX11AD69G, and NNX13AD72G (MAS), NNX10AN08A (PAC), and the Thailand Research Fund (DR). Simulations were performed at the National Center for Atmospheric Research Computational and Information System Laboratory (NCAR-CISL). The authors thank Yu. Khotyaintsev for pointing out the observations and M. M. Kuznetsova for her helpful discussion.

[1] J. Birn and E. Priest, eds., Reconnection of Magnetic Fields (Cambridge University Press, 2007).

[2] J. L. Burch and J. F. Drake, American Scientist (2009).

[3] T. E. Moore, J. L. Burch, W. S. Daughton, S. A. Fuselier, H. Hasegawa, S. M. Petrinec, and Z. Pu, JASTP, doi:10.1016/j.jastp.2012.10.004 (2012).

[4] B. U. Ö. Sonnerup, in Solar System Plasma Physics, Vol. 3, edited by L. J. Lanzerotti, C. F. Kennel, and E. N. Parker (North Halland Pub., Amsterdam, 1979) p. 46.

[5] T. Terasawa, Geophys. Res. Lett. 10, 475 (1983).

[6] M. E. Mandt, R. E. Denton, and J. F. Drake, Geophys. Res. Lett. 21, 73 (1994).

[7] M. A. Shay, J. F. Drake, R. E. Denton, and D. Biskamp, J. Geophys. Res. 103, 9165 (1998).

[8] T. Nagai, I. Shinohara, M. Fujimoto, M. Hoshino,
Y. Saito, S. Machida, and T. Mukai, J. Geophys. Res. 106, 25929 (2001).

[9] M. Oieroset, T. D. Phan, M. Fujimoto, R. P. Lin, and R. P. Lepping, Nature 412, 417 (2001).

[10] F. S. Mozer, S. D. Bale, and T. D. Phan, Phys. Rev. Lett. 89, 015002 (2002).

[11] J. D. Scudder, F. S. Mozer, N. C. Maynard, P. A. Puhlquinn, Z. W. Ma, and C. T. Russell, J. Geophys. Res. 107, 2002 (2002).

[12] A. Runov, R. Nakamura, W. Baumjohann, R. A. Treumann, T. L. Zhang, M. Volwerk, Z. Vörös, A. Balogh, K.-H. Glassmeier, B. Klecker, H. Réme, and L. Kistler, Geophys. Res. Lett. 30, 1579 (2003).

[13] F. Mozer and A. Retinó, J. Geophys. Res. 112, A10206 (2007).

[14] H. Karimabadi, D. Krauss-Varban, N. Omidi, and H. X. Vu, J. Geophys. Res. 104, 12313 (1999).

[15] M. Swisdak, J. F. Drake, M. A. Shay, and B. N. Rogers, J. Geophys. Res. 108, 1218 (2003).

[16] F. S. Mozer, P. L. Pritchett, D. S. J. Bonnell, and M. T. Chang, J. Geophys. Res. 113, A00C03 (2008).

[17] P. Pritchett, J. Geophys. Res. 113, A06210 (2008).

[18] K. G. Tanaka, A. Retinò, Y. Asano, M. Fujimoto, I. Shinohara, A. Vaivads, Y. Khotyaintsev, M. André, M. B. Bavassano-Cattaneo, S. C. Buchert, and C. J. Owen, Ann. Geophys. 26, 2471 (2008).

[19] K. Malakit, M. A. Shay, P. A. Cassak, and C. Bard, J. Geophys. Res. 115, A10223 (2010).

[20] F. S. Mozer, V. Angelopoulos, J. Bonnell, K. H. Glassmeier, and J. P. McFadden, Geophys. Res. Lett. 35, L17S04 (2008).

[21] P. A. Cassak and M. A. Shay, Phys. Plasmas 14, 102114 (2007).

[22] A. Zeiler, D. Biskamp, J. F. Drake, B. N. Rogers, M. A. Shay, and M. Scholer, J. Geophys. Res. 107, 1230 (2002).

[23] T. Lindstedt, Y. V. Khotyaintsev, A. Vaivads, M. André, R. C. Fear, B. Lavraud, S. Haaland, and C. J. Owen, Annales Geophysicae 27, 4039 (2009)

[24] P. A. Cassak and M. A. Shay, Phys. Plasmas 16, 055704 (2009).

[25] T. D. Phan and G. Paschmann, J. Geophys. Res. 101, 7801 (1996). 